\title{
Pri la genezo de la papilo de nervo optika ĉe Sus scrofa domesticus.
}

\author{
De \\ Ken Takahasi. \\ El la embriologia laboratorio en la medicina universitato de Okajama, Japanujo. \\ (P-ro J. Shikinami). \\ Kun 20 tekstofiguroj.
}

\section{Antaūparolo.}

La morfogenezo de l'okulfendo (fissura cupulae) estas unu el la plej interesaj kaj signifoplenaj aliformiĝoj en la embria evoluado de okulo, kaj havas samtempe nedisigeble intiman rilaton al la formigo de la primitiva papilo de nervo optika, tiel ke multaj aŭtoroj jam delonge diversmaniere diskutadis pri la signifo kaj formigho de l'okulfendo.

En 1921 v. Szily studis la temon el nova vidpunkto ce multaj kuniklembrioj kaj atingis novan rezultaton, ke la okulfendo aperas unue, por ke ĝi formu la primitivan papilon de nervo optika, kiel dorsa faldo ("dorsale Falte") en la spaco de l'okulventriklo ("Sehventrikel"). De antaŭ 3 jaroj mi ankaŭ esploradis pri tiu cì problemo ce kelkaj vertebruloj sub la afabla kaj entuziasma gvido de P-ro Shikinami kaj publikigis la atingitajn rezultatojn jam en Folia anatomica japonica kaj en la gazeto eldonita el Okajama medicina universitato. Tiufoje mi volas denove pritrakti pri la genezo de la papilo de nervo optika kaj la formigho de l'okulfendo por klarigi la rilaton inter ambaŭ fenomenoj ce porkernbrioj.

\section{Materialo kaj metodo.}

Ia porkembrioj uzitaj estas laūvole elektitaj el la kolektaro en nia laboratorio kaj respondas al n-ro 68-91 en la Normentafel de Keibel.

Tie ĉi mi volas esprimi mian koran dankon al P-ro Shikinami kaj D-roj Tani kaj Maruyama, kiuj delonge kun granda sindoneco 
klopodis kolekti porkembriojn por nia laboratorio, kaj afable permesis al mi uzi ilin laūvole.

El ili unuj estas fiksitaj en Zenker'a solvajo aŭ formolo kaj aliaj en Müller'a. Post peckolorigo per borakskarmino ili estas enparafinigitaj ce $56^{\circ}$ kaj seritranĉitaj laŭbezone aŭ horizontale aŭ vertikale je $10 \mu$.

Krom gipsmodeloj multaj vaksmodeloj estas kónstruitaj laŭ la metodo de Born-Peter, kaj per helpo de tiuj ĉi modeloj krom mikroskopa esplorado mi povis atingi sekvontan rezultaton.

Tabelo de la materialoj esploritaj kaj modeloj konstruitaj.

\begin{tabular}{|c|c|c|c|c|c|c|c|c|}
\hline Fazo & 1 & 2 & 3 & 4 & 5 & 6 & 7 & 8 \\
\hline $\begin{array}{l}\text { Korpolongo } \\
\text { en mm }\end{array}$ & 5,0 & 5,5 & 6,0 & 8,0 & 8,0 & 13,0 & 16,0 & 19,0 \\
\hline Tranća direkto & horiz. & horiz. & $\begin{array}{l}\text { horiz: } \\
\text { vertik. }\end{array}$ & $\begin{array}{l}\text { vertik. } \\
\text { horiz. }\end{array}$ & $\begin{array}{l}\text { vertik. } \\
\text { horiz. }\end{array}$ & horiz. & vertik. & horiz. \\
\hline Tranca diko & $10 \mu$ & $10 \mu$ & $10 u$ & $10 \psi$ & $10 \mu$ & $10 \mu$ & $10 \mu$ & $10 \mu$ \\
\hline Modelo & $100 \times$ & $100 \times$ & $100 \times$ & $100 \times$ & $100 \times$ & $100 \times$ & $100 \times$ & $100 \times$ \\
\hline Nro de embrio & Nro 55 & $\begin{array}{l}\text { Nro } 6 \\
\text { Nro } 56\end{array}$ & $\begin{array}{l}\text { Nro } 43 \\
\mathrm{~A} \& \mathrm{~B}\end{array}$ & $\begin{array}{l}\text { Nro } 42 \\
A \& B\end{array}$ & $\begin{array}{l}\text { Nro } 48 \\
\text { A \& B }\end{array}$ & Nro 50 & $\begin{array}{c}\text { nenu- } \\
\text { merita }\end{array}$ & Nro 54 \\
\hline
\end{tabular}

\section{Ĉiufaza priskribo.}

1-a fazo. (Embrio 55, kun korpolongo de 5,0 mim,

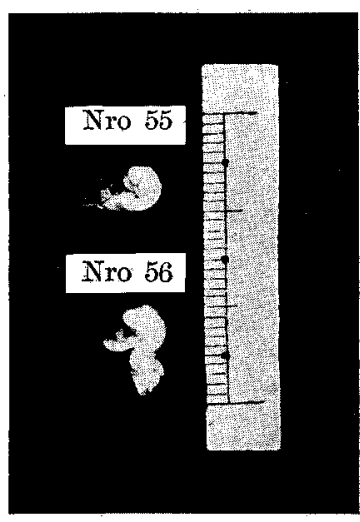

Fig. 1.

Porkembrioj 55 kaj 56 naturgrande fotografitaj. responda al n-ro 68 en la

N. T. de Keibel.)

Krom la kapoflekso estas la nukoflekso pli altgrada; la dorso kurbighas kiel arko; la vostfino staras kontră la kapo kaj turnighas dekstren; ambaŭ supraj ekstremajoj montras sin kiel tuberoj ce ŝultroregionoj; kvar brankarkoj estas kalkuleblaj.

La epitelo de la nazareo estas jam iom dika; la orelveziko kusas ce epitelo kiel pendanta guto.

En la okulo la lemsplato ("Linsenplatte") jam montras sin iom dika kaj samtempe komencas sin kavigi, por ke ĝi faru la lenssaketon en sekvonta fazo. 
Ĉe la okulveziko (vesicula optica) la laterala muro ekdikiĝas kaj samtempe ekeniras en la okulventriklon, kio unuflanke malvastigas la

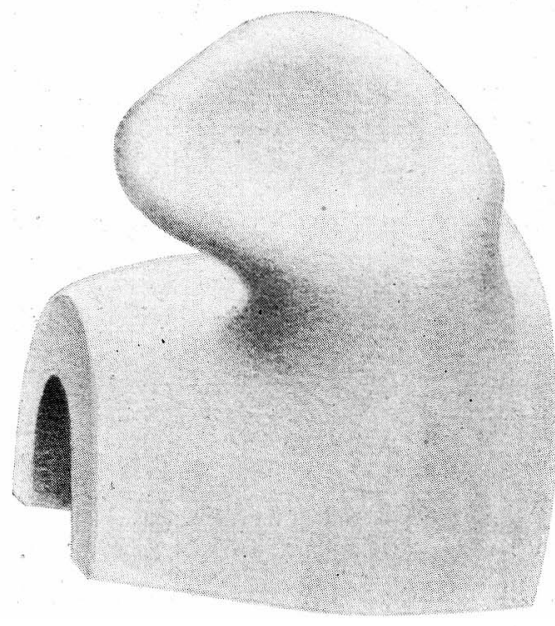

Fig. 2.

100-obligita gipsmodelo de ]'okulveziko de embrio 55, vidita de la laterala flanko. lastan kaj faras aliflanke foveeton sur la laterala muro, kaj tiamaniere komencas la transformiĝo de okulveziko al okulpokalo (cupula optica).

La okulveziko kreskas plue dorsosupren kaj tempiflanken; gia malsupra nazventra parto restas sajne sensanĝa, fiksita de relative vasta inserto de l'okulpediklo (pediculus cupulae opticae):

La okulpediklo mallonga kaj dika insertas ce la nazventra parto de l'okulveziko kaj interkomunikigas la cerban ventriklon kaj la okulventriklon per sia interua kavajo.

La okulventriklo, kiu estas parte okupita per la enirinta laterala muro de l'okulveziko, restas plejparte preskaŭ senŝanĝa kaj formas relative vastan cambron ce la inserta regiono de l'okulpediklo. Tiu cii ŝajne ne atentinda trovajo havas tamen tre gravan signifon en la plua evoluado de l'okulveziko kaj meritas rimarki.

Grandeco de la dekstra okulo elkalkulita el la mezurado ce la modelo :-

Okulveziko :-

Ekvatora cirkaŭo

Nazotempia diametro

Dorsoventra diametro

Lateralmediala diametro0,18

Okulpediklo :-

Ĉirkaŭo ce inserta regiono $\quad 0,73$

Horizontala diametro $\quad 0,28$

Vertikala diametro $\quad 0,17$

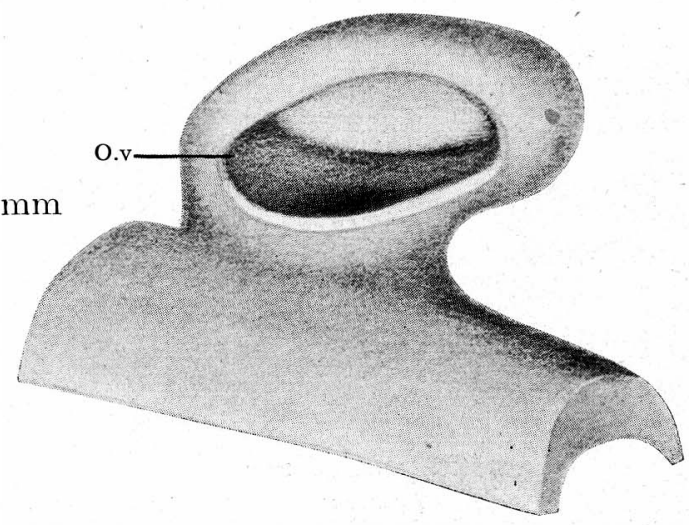

Fig. 3.

,17 La sama modelo vidita de la dorsa flanko. Ia 0,10 ekstera muro estas parte fortrancita por montri 
2-a fazo. (Fmbrio 6, kun korpolongo de $5,5 \mathrm{~mm}$, responda al n-ro 70 en la N. T. de Keibel.)

Tiu ci embrio tute similas al la embrio 56 en ekstera formo kaj en mikroskopa trovajo.

Ġ̀i montras ekstere preskaŭ nenian ŝanĝon kompare kun la antaŭa escepte de la spirala turnigro pliiĝinta ce lumbo kaj vosto, pro kio la vostfino turnas sin dorsen kaj malantaŭen.

La plidikighinta ronda nazareo plikavighas kaj formas foveon che sia centro.

La lenssaketo nun montras sin U-forma, kvankam ĝia enirejo aŭ ,, buŝo" restas ankoraŭ larĝ̀e malferma.

La transformigho de okulveziko al okulpokalo progresas iom post iom; la laterala muro (poste interna lameno) eniras pliprofunde en la okulventriklon, dum la mediala muro (poste ekstera lameno) ekstaras rekte flanken por formi hialoidan kavon (cavum hyaloideum oculi). Tiuj ci tute malaj procezoj helpas unu la alian konstrui la kompletan okulpokalon.

La spaco inter ambaŭ lamenoj, nome la okulventriklo, estas pli kaj pli okupita de la enirinta laterala nuro, por fine malaperi pro intima kontakto de ambaŭ lamenoj. Ĉe la inserta regiono de l'okulpediklo hiri tamen restas kiel restĉambro senŝanĝa ĥ̀is tiam, kiam la dorsa faldo tie ekaperos.

En tiu ći fazo ambaŭ lamenoj kuntusias tamen nenie, kvankam ili staras unu la alian tre proksime krom ce la inserta regiono de l'okulpediklo, kie la ventriklo restas ankoraŭ relative vasta, çar la transira parto de la pediklo al la okulveziko restas ŝajne senŝanĝa. Tiu ĉi fakto estas plej grava kaj signifoplena por plua morfologia evoluado de l'okulpokalo.

Grandeco de la dekstra okulo elkalkulita el la mezurado ce la modelo:-

$\begin{array}{ll}\text { Okulpokalo :- } & \\ \text { Ekratora cirkaŭo } & 1,35 \mathrm{~mm} \\ \text { Nazotempia diametro } & 0,36 \mathrm{~mm} \\ \text { Dorsoventı̈ diametro } & 0,46 \mathrm{~mm} \\ \text { Lateralmediala diametro } & 0,23 \mathrm{~mm} \\ \quad \text { Cirkaŭo de pupilrando } & 0,71 \mathrm{~mm} \\ \text { Okulpediklo :- } & \\ \text { Ĉirkaŭo ce inserta regiono } & 0,57 \mathrm{~mm} \\ \text { Horizontala diametro } & 0,27 \mathrm{~mm} \\ \text { Vertikala diametro } & 0,23 \mathrm{~mm} \\ \text { Longo } & 0,14 \mathrm{~mm}\end{array}$


Pri la genezo de la papilo de nervo optika ce Sus scrofa domesticus.

3-a fazo. (Embrio 43 A kun korpolongo de 6,0 mm kaj 43 B kun tiu de $7,5 \mathrm{~mm}$, respektive respondaj al 11 -ro $72 \mathrm{kaj}$

71. de la N. T. de Keibel.)

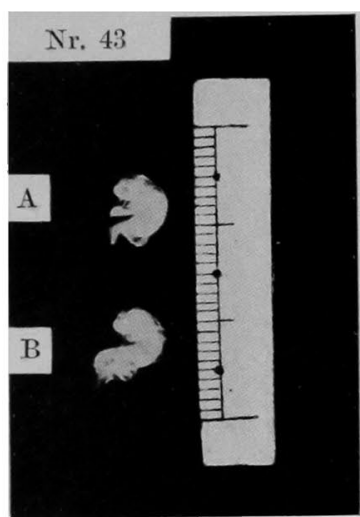

Fig. 4.

Porkembrioj 43 I kaj B, fotografitaj naturgrande.

A estas en iom pli progresinta fazo ol B; ce B la vosto turnas sin dorsen kaj malantaüen, kaŭze de la ankoraǔ altgrada spirala turnigho ĉe lumbo kaj vosto; la tuta korpo prenas tial S-formon; ce A la vosto returnas sin jam antaūen kun lumba kurbigho, sekve la korpo iom mallongigas. A estas seritrancita horizontale, $\mathrm{B}$ vertikale. La plataj braketoj kreskas laŭlonge de korpo, kaj la piedoj aperas kiel malgrandaj tubetoj $\hat{\imath}(*$ vosta parto.

$\hat{C} e^{2}$ A la naza foveo pliprofundin̂ras kaj formas nazkavon.

La lensveziko (vesicula lentis) ankoraŭ ne disiĝas de epitelo, kaj komunikas sin kun ekstera medio tra sia tre malgranda hardelikata enirejo.

La transformiĝo de okulveziko al okulpokalo estas jam fininta en tiu ci fazo, kaj la laterala muro nun fariĝas la interna lameno, dum la mediala faras la eksteran aǔ pigmentan lamenon ankoraŭ ne pigmentitan.

La okulfendo komencas sin prezenti en tiu ci fazo.

Ĉe la irado de transformigho elstaras la renversita rando de l'okulpokalo rekte flanken, kvazaŭ çi cirkaŭprenus en si la jus enirintan lenson.

Tiu ĉi procezo ne okazas samtempe cirkaŭ la tuta pupilrando, sed unue cee la dorsosupra parto, poste ice la tempia parto. La lasta klinighas kontraŭ la hialoidan kavon kaj samtempe alproksimiĝas al la naza parto, tiel ke la ventra parto, kiu lokigas inter tiuj ci du partoj kaj estas fiksita de l'okulpediklo,

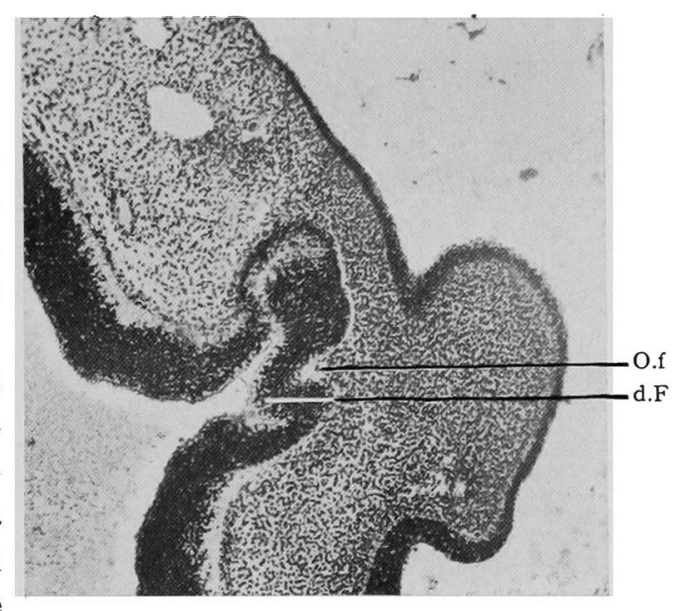

Fig. 5.

Fotografajo ( $\hat{\imath} \cdot 70$-oh)ligita) de seriero laūlonge trancita tra la regiono de l'okulfendo ie embrio 4:; 1 . O.f Okulfendo. d.f Dorsa faldo aperinta en la restíambro de l'okulventriklo. 
ŝjnas nun esti premenigita de ambaŭ partoj en la restçambron, kiu $\hat{\mathrm{g} i s}$ nun restis malplena por akcepti tiun $\hat{\mathrm{c} i}$ nove enirantan ventran muron.

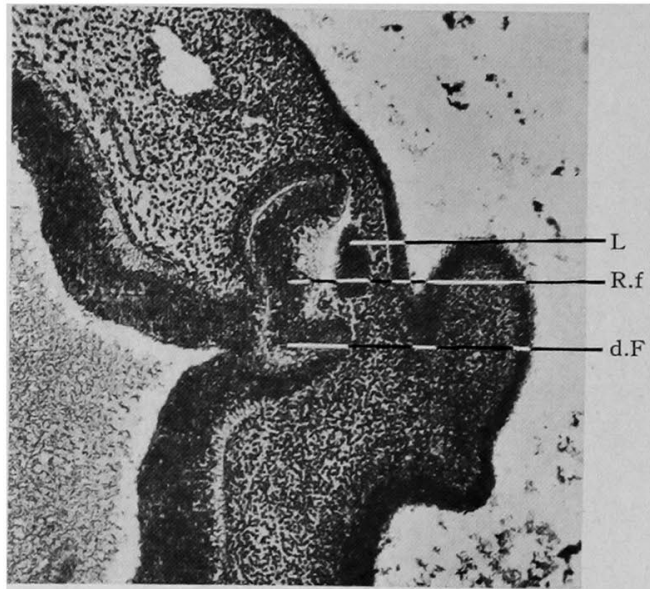

Fig. 6.

La tria seriero jo:t la antaūa montranta la retinan faldon en frua fazo. d.F Dorsa faldo. I, Lenso. X.f Retina faldo.

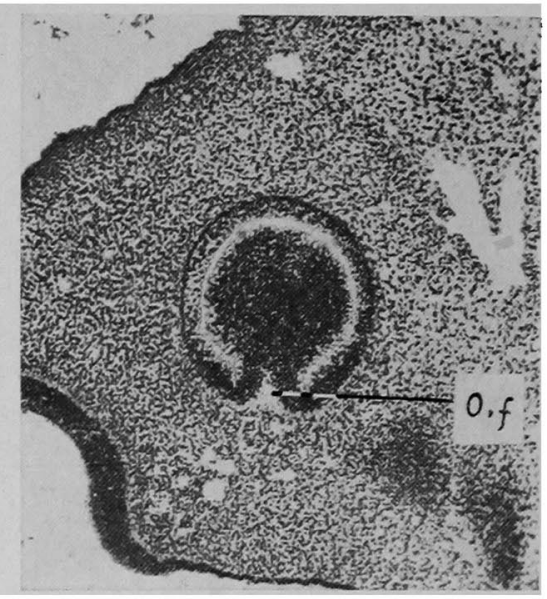

Fig. 7 .

Fotografajo (î 70-obligita) de seriero trancita vertikale tra la regiono de l'okulfendo $\hat{c}$ e la embrio $43 \mathrm{~B}$. O.f Okulfendo.

Tiamaniere ekaperas la okulfendo ce la ventra muro de l'okulpokalo, kaj kaūze de la jus enirinta ventra muro prezentiĝas nun tielnomata dorsa faldo en la restcambro. Alivorte la ekapero de l'okulfendo rekte

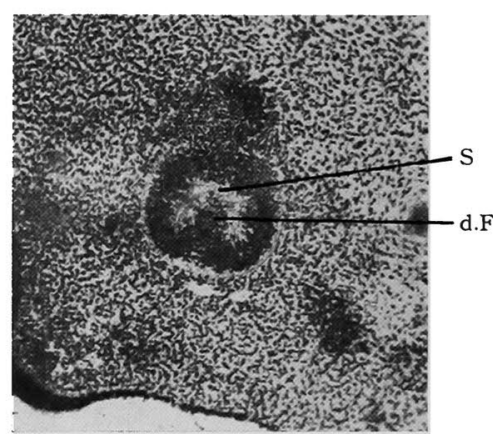

Fig. 8.

- Fotografajo de scriero trancita tra la inserta regiono de l'okulpediklo. La dorsa faldo atinginta la pediklon okupas gian tutan internan kavajon. d.F Dorsa faldo. S Postlasita malvasta spaco.

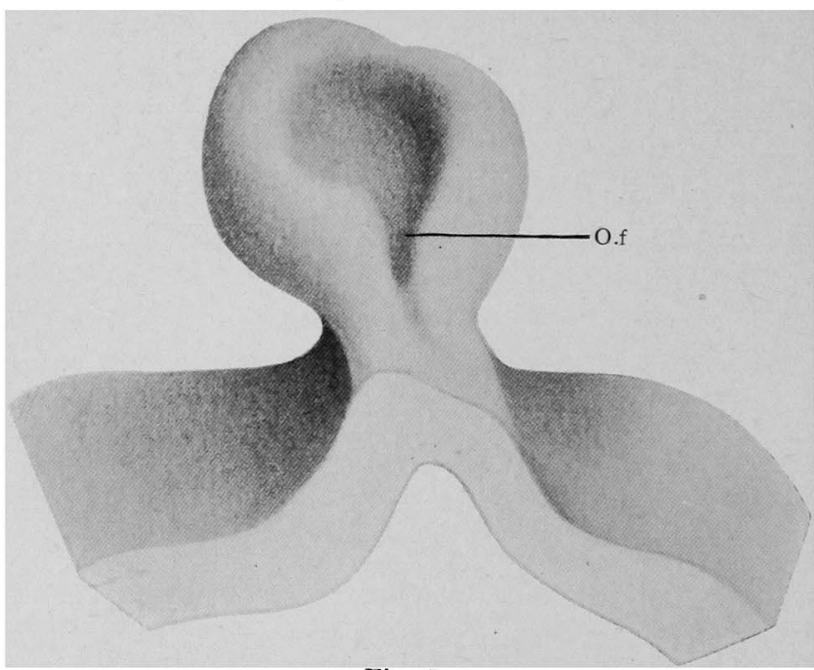

Fig. 9.

100 obligita gipsmodelo de la okulpokalo ce la embrio $4: ;$ A, vidita de la ventra flanko. O.f Okulfendo. 
Pri la genezo de la papilo de nervo optika ce Sus scrofa domesticus.

kaŭzas la naskighon de la dorsa faldo en la restêambro, kiu restis ğis c̀i tiam malplena por preti sufician spacon por $\hat{g}$ i.

Por kompreni tiun ci rilaton pli facile, oni forprenu parton de la muro ce la modelo kaj inspektu la internon de la restcambro, kie sin trovas

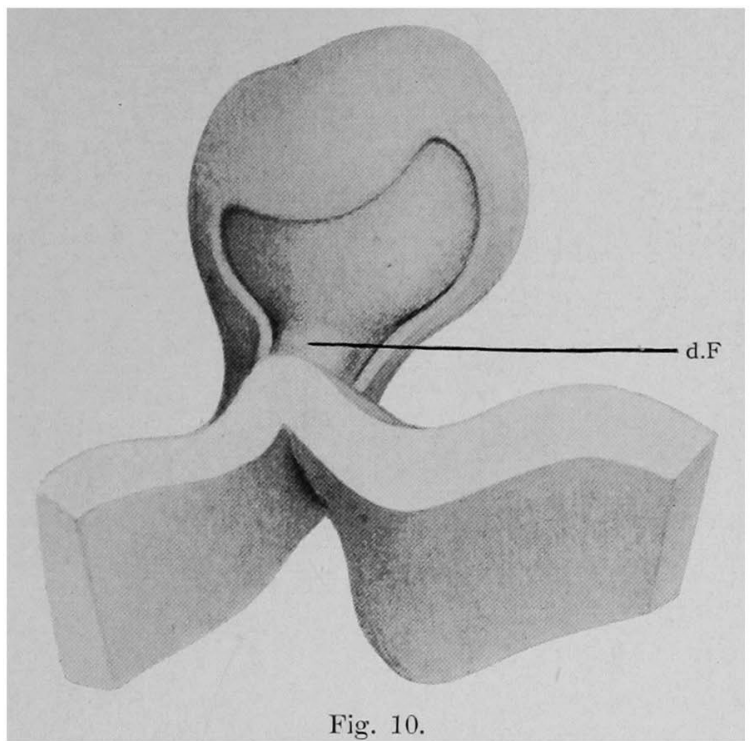

Ia sama molelo vidita de la dorsa flanko post forpreno de l'ekstera lameno, por montri la dorsan faldon. d.F Dorsa faldo. la dorsa faldo, formita de l'enirinta muro de l'okulfendo.

Tiu ci dorsa faldo estas ja la antaūajo de la papilo de nervo optika, kiu formas en plua kresko tubsimilan primitivan papilon entenantan sangvazon en sia mezo.

La dorsa faldo kuras tra la cambro, de la interna lameno al la pediklo; ĝ̀i plenigas la internan kavajon pli ol duone kaj fine $\hat{g}$ is tiom, ke tie nur plej malrasta novmondforma spaco estas postlasita. (Fig. 8.)

Se oni precize observas

la internon de la hialoida kavo, tie proksime de l'okulfendo oni trovas malaltan leviĝon de la interna lameno, kiun v. Szily unue rimarkis kiel retina faldo. Mi opinias, ke tiu $\hat{\imath} i$ retina faldo aperas pro la diferenco de kreskopovo, por ke ĝ̀i çefe subtenu la formadon de l'okulfendo.

Grandeco de la dekstra okulo (N-ro 43 A) elkalkulita el la mezurado ce la modelo :-

Okulpokalo :-

Ekvatora cirkaŭo

$1,12 \mathrm{~mm}$

Dorsoventra diametro

$0,35 \mathrm{~mm}$

Nazotempia diametro

$0,30 \mathrm{~mm}$

Lateralmediala diametro

$0,22 \mathrm{~mm}$

Ĉirkaŭo de pupilrando

$0,60 \mathrm{~mm}$

Okulpediklo :-

Ćirkaūo ce inserta regiono $\quad 0,61 \mathrm{~mm}$

Lougo

$0,16 \mathrm{~mm}$ 


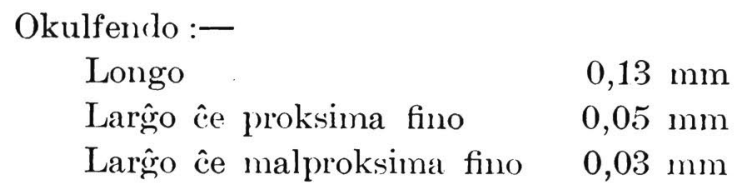

4-a fazo. (Embrioj $42 \Lambda$ kaj B kun korpolongo de 8,0 mm, respondaj al n-ro 76 de la $\mathrm{N}$. T. de Keibel.)

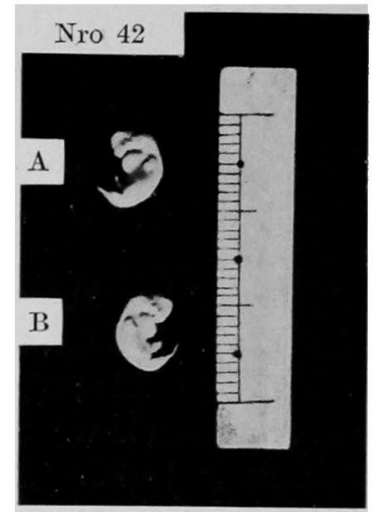

Fig. 11.

Porkembrioj t: A kaj I?, fotografitaj naturerande.
Ambaŭ estas elektitaj kiel preskaŭ egale kreskintaj; A estas trancita vertikale kaj B horizontale por observi pli precize la staton de l'okulfendo.

La cerbo kaj makzela protuberanco pligrandiGás kaj kuŝas sur la elŝ̀velinta brusto. La spirala turnigo ce lumbo kaj vosto jam malaperas kaj la pinta rostfino direktas sin antaŭen al la kapo. La braketoj fleksiĝ̣as ce kubuto kaj la plataj vermosimilaj piedoj suprenstaras kontraŭ la kapo. La nazkavo fariças pli vasta kaj pli profunda.

La lenso fermita staras ce epitelo kunigite ankoraú en punkto; ģia kavajo està tute malplena.

P'igmento ekaperas en la ekstera lameno, kaj nervofibroj sin trovas en la interna lameno.

La okulfendo longiĝninta kaj malvastiọinta restas ankoraŭ malferma en sia tuta longo, krankam siaj ambaŭ randoj en la mezo ion pli alprok:imiğas reciproke. La fendo estas tial malpli vasta en la mezo ol ce la proksima kaj malprok:ima finoj, kie la fendo estas iom pli vaste malferma kaj transiras respektive al la pediklokanelo ("Stielrimne") kaj al la pupilrando.

La pediklokanelo sin montras kiel malprofunda kaj mallonga kareto ce la ventra flanko de l'okulpediklo, sajne por facile trapasigi la hialoidan sangovazon.

En la restíanbro la dorsa faldo kuras kiel forta fasko de la retino al la pediklo, formante la pediklokanelon sur sia ventra flanko.

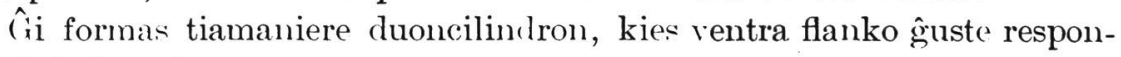
das al la kanelo mem.

En sekronta pliprogresiuta fazo la cilindrosimila dorsa faldo, manon en mano kun la fermiĝo de l'okulfendo, cirkaŭpakos la hialoidan sangvazon, kiu trairas laŭlonge de la pediklokanelo en la hialoidan 
karon, por fine formi rondan tubsimilan primitivan papilon de nervo optika.

Grandeco de la dekstra okulo (N-ro 42 B) elkalkulita el la mezurado (e) la modelo:-

$\begin{array}{ll}\text { Okulpokalo :- } & 1,24 \mathrm{~mm} \\ \text { Ekvatora cirkaŭo } & 0,37 \mathrm{~mm} \\ \text { Dorsoventra diametro } & 0,37 \mathrm{~mm} \\ \text { Nazotempia diametro } & 0,25 \mathrm{~mm} \\ \quad \text { Lateralmediala diametro } & 0,65 \mathrm{~mm} \\ \quad \text { Ĉirkaŭo de pupilrando } & \\ \text { Okulpediklo :- } & 0,62 \mathrm{~mm} \\ \quad \text { Cirkaŭo ce inserta regiono } & 0,18 \mathrm{~mm} \\ \quad \text { Longo } & \\ \text { Okulfendo kaj pediklokanelo:- } & \\ \quad \text { Longo de okulfendo } & 0,13 \mathrm{~mm} \\ \quad \text { Longe de pediklokanelo } & 0,05 \mathrm{~mm}\end{array}$

5-a fazo. (Embrioj $4 \$$ \& kaj B kun korpolongo de $s, 0 \mathrm{~mm}$, respondaj al 11-ro 78 de la N. T. de Keibel.)

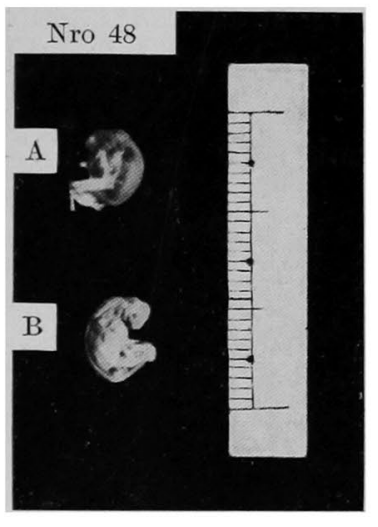

Fig. 12.

A trancita vertikale; B horizontale.

Tiuj ci embrioj kreskas iom pluen ol la antaŭaj. La cerbo kaj makzelo montras ekstere nenian rimarkindau evoluon; la mandiblo pligrandiĝ̀as.

$\hat{C}$ 'e la supraj ekstremajoj pliighas kubutflekso kaj la manoj ekaperas; ce la malsupraj ekstremajoj aperas ankaŭ genuflekso kaj la piedoj staras supren kontraŭ la kapo.

La lenso kuŝas intime ce epitclo ; ĝ̀ia malantaŭa muro ekdikiĝas por plenigi en sekvonta fazo gian kavajon per plimultighontaj fibrajoj.

Inter la lenso kaj la epitclo unuflanke kaj Porkembrioj 48 A kaj Is, la pupilrando aliflanko mezorlermêelaoj eniras: fotografitaj naturgrancle. kune kun kapilaroj, kiuj cirkaŭas la okulpokalon. Inter ambaŭ fendrandoj plilongị̂intaj sin trovas nenia diferenco; ili estas preskaŭ egale longaj kaj pli alproksimiĝa' (u la mezo, montrante la lokon de kungluigho en sekronta fazo.

Ce la proksima fino la fendo estas iom pli vaste malferma kaj transiras al la pediklokanelo, kiu nun estas pliprofunda kaj preta ĉirkaŭpaki la sangrazon enirantan cn la hialoidan kavon. 
La formo de la pupilo ne estas roncla sed elipsa; ýia rando staras ne sur sama ebenajo pro la malegaleco de kreskopovo. La okulpokalo kreskas nome pli forte en dorsosupra kaj tempia partoj; la respondaj randpartoj pligrandighas kaj plidikighas ol aliaj, farante tiamaniere incizurojn aŭ "Einkerbungen".

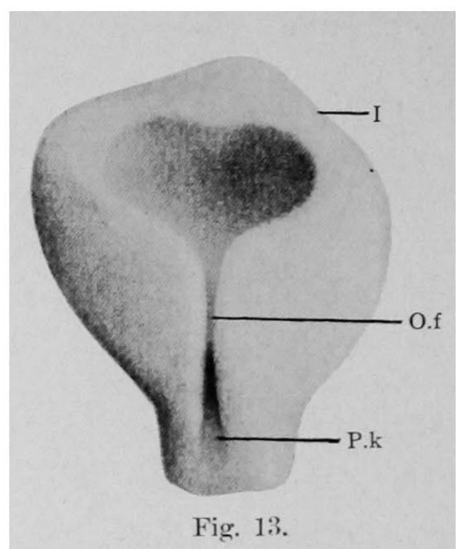

100 obligita gipsmodelo de l'okulpokalo de embrio tis 1 , montranta la okulfendon fermityontan en la mezo. Vidita de la rentra flanko. ().f Okulfendo. P.k Pediklokanelı. I Incizuro.

Per forpreno de parto de l'ekstera lameno cee la modelo oni trovas interne la dorsan faldon kurantan de la interna lameno al la pediklo tra la restçambro de l'okulventriklo.

La dorsa faldo catas nun tiel dika kaj forta, ke k̂̀i okupas la tutan restêambron en formo de duoncilindro aŭ fasko; la pediklokanelo iom profundignass ce sia ventra flanko kaj sempere transiras al la okulfendo.

La pediklokanelo śajnas ekstere tute aparteni al la pediklo mem; sed oni povas facile kompreni, ke estas la dora faldo, kaj ne la pediklo mem, kiu montras la pediklokanelon ce sia ventra flanko. Oni povas ankaú imani la formon de la dorsa faldo kiel duonigita sielumo, kies kavajo respondas al la pediklokanelo.

Tiamaniere la fermigho de la pediklo-

kanclo rekte signifas la fermighon do la dorsa faldo mem cirkaĭ la pediklokanelo, kio okazas tuj post la fermigho de l'okulfendo.

Grandeco de la dekstra okulo (N-ro 49 A) elkalkulita el la mezuro ie la modelo :-

Okulpokalo:-

Ekvatora cirkaŭo

Dorsoventra diametro

Nazotempia diametro

$1,08 \mathrm{~mm}$

$0,32 \mathrm{~mm}$

Lateralmediala diametro

$0,40 \mathrm{~mm}$

$0,26 \mathrm{~mm}$

C'irkaūo de pupilrando

$0,64 \mathrm{~mm}$

Horizontala diametro

Tertikala diametro

$0,22 \mathrm{~mm}$

$0,17 \mathrm{~mm}$

Okulpediklo :-

Ĉirkaūo te inserta regiono

Longo

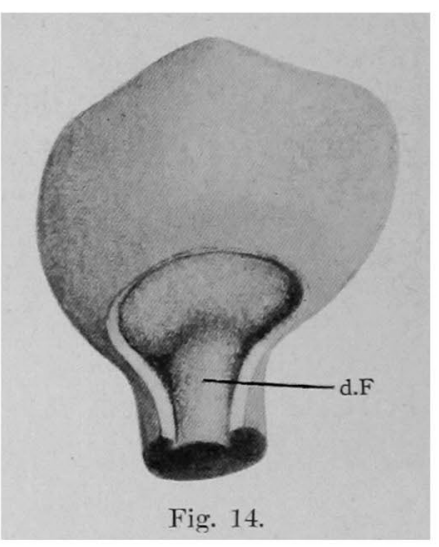

Ia sama modelo vidita de la dorsa flanko post forpreno de parto de l'ekstera lameno kaj de la dorsa muro de pediklo. Oni vidas, kiel la dorsa faldo kuniga: retinon kaj pediklon. d.F Dorsa faldo. 
Pri la genezo de la papilo de nervo optika ce sus scrofa domesticus.

Okulfendo kaj pediklokanelo :-
Longo de okulfendo
$0,17 \mathrm{~mm}$
Longo de pediklokanelo
$0,06 \mathrm{~mm}$

Grandeco de la dekstra okulo (Nro 48 B) elkalkulita el la mezuro ce la modelo :-

$\begin{array}{ll}\text { Okulpokalo :- } & \\ \text { Ekvatora cirkaŭo } & 1,25 \mathrm{~mm} \\ \text { Dorsoventra diametro } & 0,39 \mathrm{~mm} \\ \text { Nazotempia diametro } & 0,37 \mathrm{~mm} \\ \quad \text { Lateromediala diametro } & 0,24 \mathrm{~mm} \\ \quad \text { Ĉirkaūo de pupilrando } & 0,72 \mathrm{~mm} \\ \text { Okulpediklo :- } & \\ \quad \text { Ĉirkaŭo ce inserta regiono } & 0,42 \mathrm{~mm} \\ \quad \text { Longo } & 0,20 \mathrm{~mm} \\ \text { Okulfendo kaj pediklokanelo :-- } & \\ \quad \text { Longo de okulfendo } & 0,15 \mathrm{~mm} \\ \quad \text { Longo de pediklokanelo } & 0,05 \mathrm{~mm}\end{array}$

6-a fazo. (Embrio 50, kun korpolongo de $13 \mathrm{~mm}$, responda al n-ro 83 en la N. T. de Keibel).

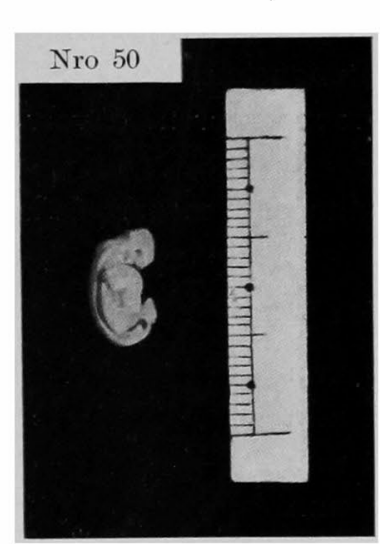

Fig. 15.

Tiu ĉi 'embrio estas en pli kreskinta fazo, precipe en kapo kaj ventro. La cerbo estas pligranda; la makzelo kaj mandiblo estas ankaŭ plikreskintaj kaj formas buŝn. La vosto komencas envolviĝ̀i kontraŭ la ventro.

La lenso kuŝas ankoraŭ intime ce epitelo; gì kavajo estas gis malgranda novmondforma spaco plenigita de ciam plimultiĝantaj lensfibroj.

Mezodermceloj kaj kapilaroj, kiuj sin trovas ĉirkaŭ la okulpokalo, enmigras unuflanke en la spacon inter lenso kaj epitelo kaj aliflanke tra la spaco inter lenso kaj pupilrando en la hialoidan kavon, kie la kapilaroj formas kapilarreton kun Porkembrio 5 ) fotografita naturgrande. la hialoidaj sangovazoj.

En tiu ci fazo la okulfendo intime kuntuŝas en la mezo, levante ambaŭ randojn en la hialoidan kavon, kio rememoriGas nin je la fermado de l'okulfendo ce birdembrio.

La proksima kaj malproksima finoj de la fendo restas tamen ankoraŭ 
malfermaj; la unua senpere transiras al la pediklokanelo, kiu farighas iom post ion profunda kaj samtempe tub-aŭ î̀lumsimila, entenante en si la hialoidan sangvazon, dum la malproksima fino de la fendo transiras al la pupilrando.

Per la figuroj oni povas facile kompreni, kiamaniere ambaŭ randoj de la fendo reciproke alproksimigas unue en la mezo, çis ili kuntuŝas kaj kungluiĝas por fine komplete fermiĝi.

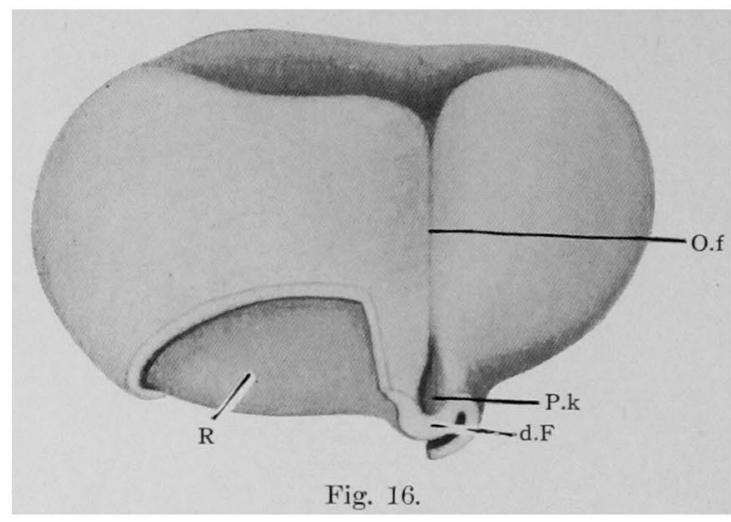

100-obligita gipsmodelo de l'okulpokalo ce embrio 50. I a okulfendo intime kuntusas en la mezo kaj ambaū finoj transiras respektive al la pediklokanelo kaj al la pupilrando. O.f Okulfendo kungluighinta en la mezo. P.k Pediklokanelo kun proksima fino de l'okulfendo. li Retino. d.F Dorsa faldo.
Tuj post la kompleta fermiĝo de la fendo ambaŭ lamenoj apartighas ce la fermita loko denove en internan kaj eksteran' lamenojn, el kiuj la unua restas tie kiel retina faldo çi ankoraŭ progresinta fazo, montrante la fermitan lokon de l'okulfendo, dume la lasta postlasas tie nenian signon, escepte de malmulte da pigmento, kiu tuj farighas nekonebla. Kiel la figuro 16 aŭ 19 montras, la proksima fino de la fendo formas

kune kun la pediklokanelo mallongan kaj malvastan trueton, tra kiu la hialoilla sangrazo kuras, kaj kiu sin fermas desupre malsupren, envolyante la sangrazon de ambaŭ flankoj.

Grandeco de la dekstra okulo (Nro 50) elkalkulita el la mezuro ĉe la modelo :-

$\begin{array}{ll}\text { Okulpokalo :- } & \\ \text { Ekvatora cirkaŭo } & 1,89 \mathrm{~mm} \\ \text { Dorsoventra diametro } & 0,54 \mathrm{~mm} \\ \text { Nazotempia diametro } & 0,63 \mathrm{~mm} \\ \text { Laiteralınediala diametro } & 0,34 \mathrm{~mm} \\ \text { Cirkaŭo de la pupilrando } & 1,15 \mathrm{~mm} \\ \text { Horizontala diametro } & 0,36 \mathrm{~mm} \\ \text { Vertikala diametro } & 0,30 \mathrm{~mm}\end{array}$

Okulpediklo :-

Ĉirkaŭo $\hat{\imath} e$ inserta regiono $0,37 \mathrm{~mm}$

Longo

$0,32 \mathrm{~mm}$ 
Pri la genezo de la papilo de nervo optika ce Sus scrofa domesticus.

Okulfendo kaj pediklokanelo :-

Longo de malferma parto de okulfendo ce pupilrando $\quad 0,04 \mathrm{~mm}$

Longo de fermita parto de okulfendo en la mezo $\quad 0,18 \mathrm{~mm}$

Longo de pediklokanelo $\quad 0,06 \mathrm{~mm}$

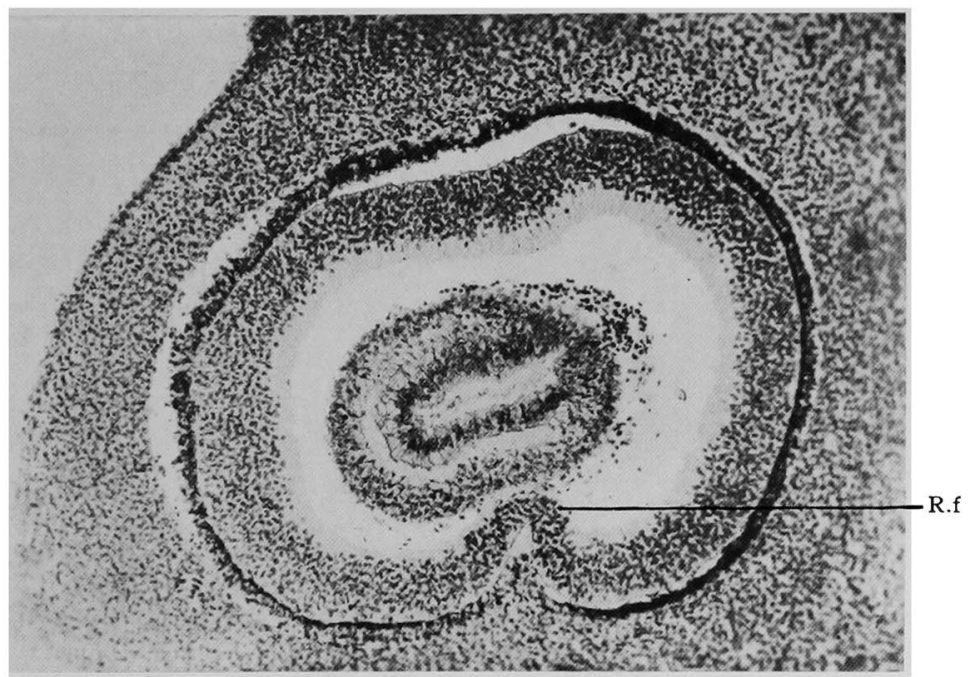

Fig. 17.

Retina faldo ce la fermita parto de l'okulfendo. R.f Retina faldo.

7-a fazo. (Embrio nenumerita, kun korpolongo do $16 \mathrm{~mm}$, responda al n-ro 88 en la $\mathrm{N}$. T. de Keibel.)

Tiu ĉi trançoserio estas farita en Germanujo de P-ro J. Shikinami, kiu grin afable pruntedonis al mi por kompara studio. Fotografajo de la tuta korpo ne estis havebla.

La lenso estas num kompleta ; ie la korneo ciuj tavoloj estas distingeblaj. La antaǔcambro ankoraǔ ne aperas, sed tra la interspaco inter korneo kaj lenso kuras maldika delikata kapilarmembrano, kiu enpakas la lenson kune kun kapilarreto en la vitra korpo (corpus vitreum). La iriso estas ankoraŭ ne ekkonebla, kvankam maldensa mezodermćclaro, kiu sintrovas cirkaŭ la pupilrando kaj enhavas en si tielnomatan grandan arterian ringon, indikas venontan disvolvighon de l'iriso. En tiu ĉi fazo la okulfendo fermas perfekte es la mezo, kaj tie la retino sin montras kiel faldo (fig. 17). 
$\hat{C}$ e la pupilrando kaj ce la pediklokanelo restas la fendo ankoraŭ malferma, kaj mezurighas $0,08 \mathrm{~mm}$ kaj $0,1 \mathrm{~mm}$ longa respektive. Će la proksima fino la fendo formas kune kun la pediklokanelo malgrandan trueton fistulsimilan, kies dorsa flanko estas limita de la dorsa faldo, dum gịa ventra limo estas farita de ambaŭ fendrandoj kungluighintaj. La fermiĝ́o de la pediklokanelo marŝas kun tiu de la fendo, de la malproksima parto al la proksima, kovrante la sangvazon de ambaŭflankoj, por fine postlasi tre malgrandan enirejon por la eniranta sangvazo.

Tiamaniere la dorsa faldo farighas fine, post kompleta fermigho de la pediklokanelo, tubo- aü cilindrosimila primitiva papilo enhavanta fistulon, tra kiu la sangrazo atingas la hialoidan kavon.

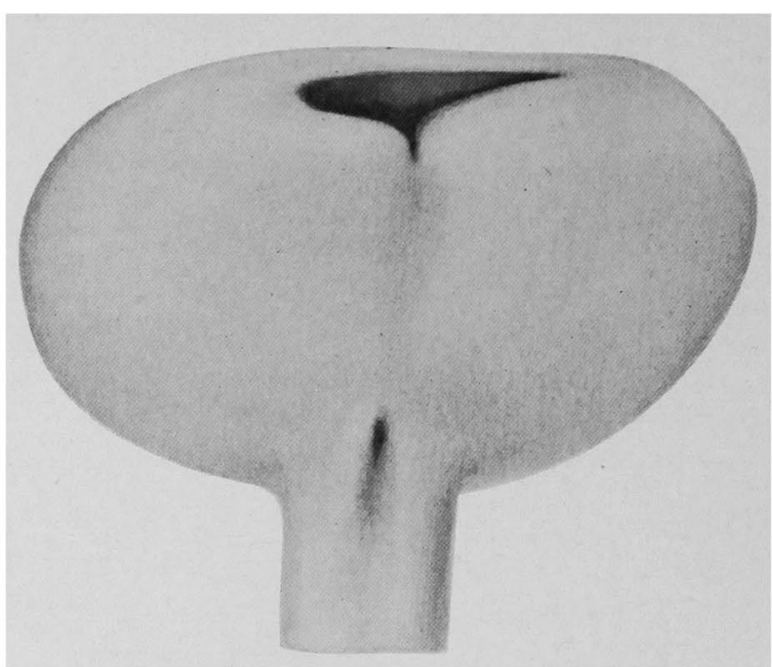

Fị. 1S.

100 obligita modelo de l'okulpokalo $\hat{\text { ce }}$ l'embrio nenumerita. I.a okulfendo estas jam perfekte fermita en la mezo.
Kelkajn vortojn pri la retina faldo. v. Szily unue rimarkis pri la faldo, kiu aperas en relative frua fazo de la embria okulo. Ii rliras, ke gi aperas, por ke ĝi helpu la formighon de l'okulfendo, çar ĝi ekaperas jus antaŭ ĝia formigro kaj malaperas tuj post ríia formigno. Mi ankaū povis trovi la retinan faldon en la 3-a fazo (fig. 6), (n kiu la okulfendo estas formita. Oni tamen observas duan retinan faldon en pli malfrua fazo, en kiu la okulfendo estas jam fermita. Ĝi postsignas la fermitan fendlokon kaj restas k̂is ankoraŭ pli progresinta fazo. Tiuj ‘i du retinaj faldoj devas prezenti tial tute malsamajn formajojn, kvankam same nomitaj.

Grandeco de la dekstra okulo elkalkulita el la mezuro ce la modelo. Okulpokalo :-

$\begin{array}{ll}\text { Ekvatora c̈irkaŭo } & 2,20 \mathrm{~mm} \\ \text { Dorsoventra diametro } & 0,(5.5 \mathrm{~mm} \\ \text { Nazotempia diametro } & 0,80 \mathrm{~mm} \\ \text { Lateralmediala diametro } & 0,45 \mathrm{~mm}\end{array}$

$2,20 \mathrm{~mm}$

$0,80 \mathrm{~mm}$

$0,45 \mathrm{~mm}$ 
Pri la gene $\%$ de la papilo de nervo optika îe sus scrofa domesticus.

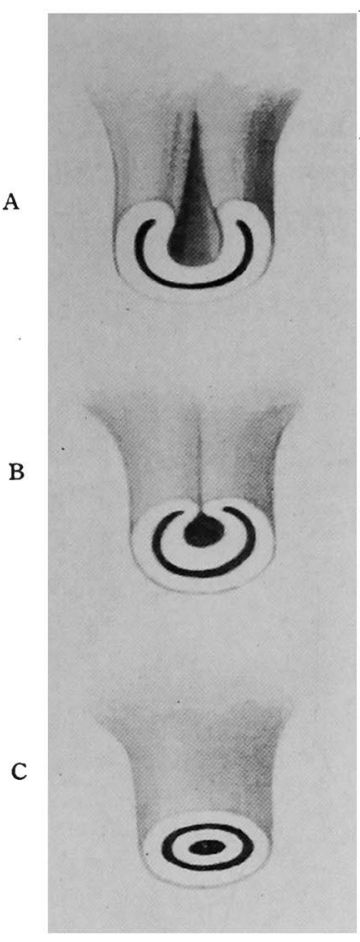

Fig. 19.

Fermomaniero de la pediklokanelo, iom trograndigite desegnita por facila kompreno.

A Il la fermigho de l'okulfenclo $\sin$ sekvas tiu de l'pediklokanelo.

13 La fermiĝ́o de la pediklokanelo estas jus fininta, cirkaūpakante la trairejon de l'sangvazo.

(. Tuj post la kompleta fermiôn apartiôas: la interna kaj ekstera lamenoj unu de la alia; la interna lameno jam formas rondan primitivan papilon de nervo optika.
Ćirkaŭo de pupilrando

$1,40 \mathrm{~mm}$

Horizontala diametro

(),38 $\mathrm{mm}$

Vertikala diametro

$0,32 \mathrm{~mm}$

Okulpediklo :-

Ĉirkaŭo ce inserta regiono

$0,40 \mathrm{~mm}$

Longo

$0,32 \mathrm{~mm}$

Okulfendo kaj pediklokanelo:-

Longo de malferma parto de okulfendo će pupilrando

$0,0 \mathrm{smm}$

Longo de fermita parto de okulfendo ce meza parto $\quad 0,26 \mathrm{~mm}$

Longo de pediklokanelo kun proksima fino de okulfendo $0,10 \mathrm{~mm}$

8-a fazo. (Embrio 54, kun korpolongo de $19 \mathrm{~mm}$, responda al n-ro 91 en la N. T. de Keibel).

La disvolvigho estas en ciuj partoj rimarkinda, la kapo grandiĝinta, la ventro elŝvelinta kaj la kvar ekstremajoj kreskintaj tute atingas la kompletan formon de porkembrio.

$\hat{C}$ e la okulo ambaŭ palpebroj aperas kiel kolbasoj kaj limas inter si palpebran fendon.

La korneo konsistas el jenaj tavoloj: Epitelo konsistanta el du vicoj de plataj celoj, tre maldika Bowman'a membrano, propra substanco, kiu okupas plejparte da korneo, Descemet'a membrano kaj endotelo.

La lenso estas nun ronda globo korrita de kapilarreto kaj estas plenigita de lensfibrajoj.

Naldensa mezodermcelaro enhavanta la grandan arterian ringon sin trovas cirkaŭ la pupilrando kiel antaŭaĵo aŭ anlago de l'iriso, de kie kapilaroj enmigras en la hialoidan kavon.

En la retino oni povas klare distingi nervofibran kaj celan tavolojn, kaj kiel postsigno de la fermita okulfendo la retinan faldon.

Tiu cii faldo estas kompreneble malsama de tiu retina faldo, kiu aperis en pli frua fazo, ce la formigho de la okulfendo.

En tiu çi fazo la okulfendo kaj pediklokanelo estas perfekte 
fermitaj, kaj jen la ronda papilo de nervo optika enhavanta sangvazon en la centro estas jam kompleta.

La dorsa faldo, kiu aperis kiel antaŭajo de la papilo, iom post iom cirkaŭas la pediklokanelon kaj formas fine kun la

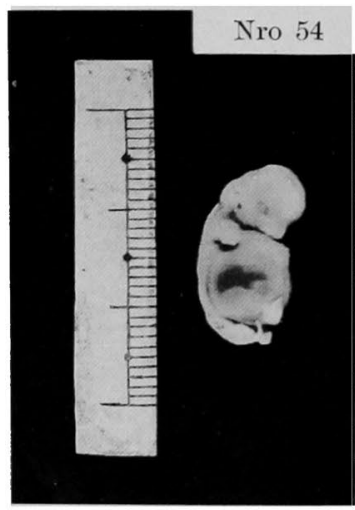

Fig. :2).

Porkembrio 54 fotografita naturgrande. kompleta fermigho de la fendo kaj pediklokanelo la primitivan tubsimilan papilon de nervo optika.

Grandeco de la dekstra okulo elkalkulita el la mezuro ìe la modelo :-
Okulpokalo :-
Ekvatora cirkaŭo
$2,75 \mathrm{~mm}$
Dorsoventra diametro
$0,73 \mathrm{~mm}$
Nazotempia diametro
$1,00 \mathrm{~mm}$
Lateralmediala diametro
$0,67 \mathrm{~mm}$
Cirkaūo de pupilrando
$1,72 \mathrm{~mm}$
Horizontala liametro
(), $55 \mathrm{~mm}$
V'ertikala diametro
$0,4 \mathrm{smm}$
Okulpediklo :-
Cirkaūo ce inserta regiono $\quad 0,35 \mathrm{~mm}$
Longo
0,40 $\mathrm{mm}$.

\section{Resumo kaj konkludo.}

Kiel jam en la ciufaza priskribo dirite, la ,, anlago" de la papilo de nervo optika aperas kiel dorwal faldo en la restêmbro de l'okulventriklo, kiam la transformigho de okulveziko al okulpokalo estas fininta kaj la okulfendo jus aperis $\hat{\imath}$ e gía ventra muro.

Konforme al la kreskidlo de okulpokalo la okulfendo longiğas pli kaj pli lateralen, por plenformi la ventran muron de l'okulpokalo.

La dorsa faldo kreskas ankaŭ en la restíambro kiel dika fasko, kiu ludas rolon por unuigi aŭ, plibone dirite, transkunligi la retinon kaj pediklon.

Kun plilongiĝ́o de l'okulfendo ckaperas ĉe la ventra muro de la pediklo tielnomata pediklokanelo, kiu kunigas sin per sia malproksima fino kun la okulfendo kaj helpas facilan trapason de la hialoida sangvazo. Ekstere la pediklokanelo sajnas aparteni al la pediklo mem, per interna inspekto oni tamen povas facile kompreni, ke gi g. guste formas la ventran muron de la clorsa falilo. La lasta faras nome duoncilindron, kies ventran muron okupas la pediklokanelo.

Ĉe nia embrio 50 la okulfendo komencas fermi en la mezo, kie ambaŭ fendrandoj intime kuntuŝas, levante sin kiel faldoj en la hialoidan 
kavon. La fermiĝo de la fendo marŝas de tie al ambaŭ finoj proksima kaj malproksima; la procezo transiras iom post iom al la pediklokanelo, kiu fariộas nun pli profunda kaj estas preta sinfermi, alproksimigante siajn randojn por enpaki la sangvazon, kiu trapasas la pediklokanelon.

Kun kompleta fermiğo de la fendo la pediklokanelo ankaŭ sin fermas demalproksime proksimen (če la bildo desupre malsupren), entenante la sangvazou en la centro.

Tuj post la kompleta fermiĝo ambaŭ lamenoj interna kaj ekstera apartiĝas unu de la alia; en la okulpokalo la interna lameno postsignas la fendlokon kiel retina faldo, kaj ce la inserta regiono la dorsa faldo nun perfekte cirkaŭas la sangvazon kaj faras mallongan tubeton. La primitiva papilo de nerva optika estas tiamaniere formita kaj tre malgranda enirejo por la sangvazo restas fine kiel la lasta postsigno de la pediklokanelo.

Ce nia embrio 54, kiu respondas al n-ro 91 de la N. T. de Keibel, la fermiĝo de l'okulfendo kaj pediklokanelo estas jam fininta, kaj la papilo de nervo optika estas ankaū perfekte formita. Jen estas la atingitaj konkludoj :

La apero de l'okulfendo kaŭzas la formiĝon de la dorsa faldo en la restcambro de l'okulventriklo. La okulventriklo restas ce la inserta parto de l'okulpediklo kiel restîambro tiom sufice vasta, ke la dorsa faldo povas evolui pluen. La dorsa faldo kreskas pluen kiel fasko aŭ cilindro kunliganta la retinon kaj pediklon, kaj post kompleta fermigho de l'okulfendo ĝi ankaŭ fine fermighas cirkaŭ la pediklokanelo aŭ ĝustedire cirkaŭ la hialoida sangvazo, formante tiamaniere la tubsimilan primitivan papilon de nervo optika. La pediklokanelo aperas, por ke la dorsa faldo cirkaŭprenu la sangvazon kaj samtempe formu la cilindrosimilan papilon.

Fine mi volas esprimi mian koran dankon al P-ro J. Shikinami, kiu min vigligis kaj gvidis en la laboro, kaj santempe al P-ro S. Nisi, kiu afable prenis sur sin la korektadon de la skribajo. 


\section{Ĉefa literaturo.}

1) Alexander, J., Zur Entwicklungsgeschichte des Wirbeltierauges. Anat. Anz. Bd. 51. 1918.

2) Baltelmez, G. W., The origin of the optic and optic primordia in man. Journ. comp. neur. 34. 192:.

3) Beckwith, C. J., The effect of the eye in Amblystoma punctatum, with special reference to the choroid fissure. The Journ. of Expr. Zool. Vol. 49. 1927.

4) Deyl, J., Über den Eintritt der Art. cent. retinae in den Sehnerv beim Menschen. Inat. Anz. Bd. 11. 1896.

5) Ellenberger, W. u. Baum, H., Handbuch der vergleichenden Anatomie der Haustiere, Berlin 1921.

6) Fessler, F., Zur Entwicklungsmechanik des Auges. Arch. f. Entwicklungsmechanik. Bi. 46, 19:0.

7) Fracassi, G., Entwicklung u. Morphologie des Glaskörpers beim Menschen und einigen säugetiere. Graefe Arch. 111. 19:3.

8) - - Bemerkungen zur Embryologie des Auges. Graefes Arch. 115. 1925.

(1) Franz, V., Studien zur vergl. Anat, d. Säugetiere. Arch. f. vergl. Opthalmol. 1. 1910.

10) Froriep, 1., Die Entwickelung des Auges der Wirbeltiere. O. Hertwig, Handbuch d. vergl. u. experim. Entwickiungsgeschichte. 2, 2. 1905.

11) Fujii, K., Über die Morphogenese eines aus Tervenfasern bestehenden Stranges, der sich im Glaskörper des abnormen Auges beim Kaninchen befindet. Xihon Gankagakkai Zasishi 30, 1926.

12) Fuchs, H., Zur Entwicklungsgeschichte des Wirbeltierauges, über d. Entwicklung d. Augengefässe des Kaninchens. Anat. Hefte 28, 1905.

13) Glückmann, A., Zur Entwicklung der vorderen Augenkammer, des Glaskörpers und Hornhaut beim Menschen usw. Zeitschr. f. Anat. u. Entwickl. Bd. 88, 1920.

14) Hagedorn, A., The early development of the endothelium of Descemet membrane, the cornea and the anterior chamber of the eye. British Journ. Ophth. 12, 1928.

15) Hertwig, O., Lehrbuch der Entwicklung*geschichte des Menschen und der Wirbel tiere. 10. Aufl. 1915.

16) Jokl, A, Über den Verschluss der fötalen Augenbecherspalte usw. im embryonalen Reptilienauge. Zeitschr. f. Anat. u. Entwicklg. Bd. 68. 1923.

17) Keibel, F., Nomentafel zur Entwicklungsceschichte des schweines. Jena. 1897.

18) -, Entwicklung des Wirbeltierauges. Klin. Mbl. d. Augenheilkunde. 44. 1906.

19) Keibel, F. u. Iall, F. P., Handbuch der Entwicklungsgeschichte des Menschen. J.eipzig, 1911.

20) Keil, R., Beiträge zur Entwicklungsgeschichte des Auges vom Schwein mit besonderer Berücksichtigung des Verhaltens der fïtalen Augenbecherspalte. Inat. Hefte :3:, 1907.

21) Lindahl, C., Über die Pupillaröffnung des Augenbechers in früheren Entwicklstd. usw. Arch. f. Augenhailk. 72, 191:.

22) Lindahl, C. u. Jokl, A., Über den Verschluss der fötalen Augenbecherspalte, die Entwicklung des Sehnerveninsertion und die Anlage des Pectens bei Vögeln. Zeitschr. f. Anat. u. Entwicklungsgeschichte. Bd. 63, 1922. 
Pri la genezo de la papilo de nervo optika ce Sus scrofa domesticus.

23) Iann, I. C., The relations of the hyaloidcanal in the foetus and Adult. Journ. of anat. 62,1928 .

24) Nussbaum, M., Entwicklungsgeschichte des menschlichen Auges. Graefe-Saemisch Handb. d. ges. Augenheilk. 1900.

25) Rabl, C., Über die bilaterale und nasotemporale Symmetrie des Wirbeltierauges. Arch. f. mikroskop. Inat. 90, Abt. 1, 1917.

26) Seefelder, R., Beiträge zur Entwicklung des menschlichen Auges mit besonderer Berücksichtigung des Verschlusses der fötalen Augenspalte. Anat. Hefte, Bd. 48, 1913.

27) —_- Über die Faltenbildung der embryonalen Retina. Graefes Arch. 111, 1923.

28) Suganuma, S., Ửber die Fntwicklung des Glaskörpers. Tyûgai-ijisinpô, nro 678, 1908.

29) Spemann, H., Zur Entwickiung des Wirbeltierauges. Zool. Jahrb. 32, 1912.

30) v. Szily, A., Das Problem der Augenbecherspalte und die Entstehung der Papiila nervi optici beim Kaninchen. Graefes Arch. 106, 1921.

31) - Morphogenese des Sehnerveneintrittes und des Fächers beim Hühnchen. Graefes Arch. 107, 1922.

32) Takahashi, K., Über den Verschluss der fötalen Augenbecherspalte bei Uroloncha domestica Flower. Med. Universität Okayama, Bd. 2. 1930.

33) — - Über die Morphogenese dên fötalen Augenbecherspalte bei Megalobatrachus japonicus. Folia Anat. Japon. Bd. 8, 1930.

34) Wolf rum, C., Multiple Einkerbungen des Becherrandes der sekundäre Augenblase usw. Klin. Mbl. f. Augenheilk. 46, 1908. 